\title{
Characterisation of silicone prepolymers and disparity in results
}

\author{
G. B. Shah* \\ Applied Chemistry Laboratories (ACL), PINSTECH P. O. Nilore, Islamabad, Pakistan
}

Received 11 September 2008; accepted in revised form 17 October 2008

\begin{abstract}
Liquid hydroxyfunctional polydimethylsiloxanes prepolymers (HOPDMS, Siloprens) with a range of relative molar masses, $M_{n}(25500-88000 \mathrm{~g} / \mathrm{mol})$ quoted by the supplier have been characterised by a number of techniques such as $\mathrm{OH}$ group titration, bulk viscometry, Gel Permeation Chromatography (GPC), and swelling measurements. The resulting molar masses of these prepolymer obtained through these techniques have been compared with one another and that of the values quoted by the manufacturer. Unexpected results of insensitivity of the properties of these prepolymer to the initial molar masses quoted by the suppliers have been observed. It has been found out that these results are dependent upon the type of technique used. The reason for these unexpected results has been discussed in detail and attributed to the multi functionality of the prepolymers, a characteristic not previously suspected or reported.
\end{abstract}

Keywords: material testing, prepolymers, viscosity average molecular weight, number average molecular weight, molecular weight between crosslinks

\section{Introduction}

Polymers and prepolymers are usually characterised with a wide range of techniques. These techniques range from molecular weight determination to analytical methods. The molecular weight of a polymer or prepolymer quoted in a different form is dependent upon the techniques used for its determination. Among them the most likely quoted are the number average molecular weight $\left(M_{n}\right)$ and weight average molecular weight $\left(M_{w}\right)$ while a third type of molecular weight referred occasionally is designated as z-average molar mass $\left(M_{z}\right)$.

Usually $M_{w}$ is greater than the $M_{n}$ except for an ideal system where uniform molar mass $\left(M_{w}=M_{n}\right)$ is concerned. This difference in the values of $M_{w}$ and $M_{z}$ leads to the spread of molar masses i.e. dispersivity $\left(M_{w} / M_{n}>1\right)$. It is an established fact that $M_{w}$ is sensitive to high molar masses while the $M_{n}$ is affected more with the lower molecular weight. There are quite a large number of methods [1] for determining the average molar mass of a polymer but relatively few for investigating the distribution of that molar mass. The knowledge of the poly dispersivity of a polymer is important to understand the variation in its processing conditions [2-4] and subsequently help in tailoring it according to the requirements.

In the present study liquid hydroxyfunctional polydimethylsiloxane prepolymers having different molar masses (quoted by the manufacturers) were characterised with different techniques and the respective results are compared with one another. The poly dispersivity of the polymer with respect to different techniques has been discussed. 


\section{Experimental part}

\subsection{Materials}

The liquid hydroxyfunctional polydimethylsiloxanes (HOPDMS) prepolymers used were Silopren C1, C2, C5, C18, and C50 supplied by Bayer Ltd. Their respective molecular weights (relative molar masses) $\left(M_{n}[\mathrm{~g} / \mathrm{mol}]\right)$ quoted by the manufacturer [5] were 25500 (C1), 34500 (C2), 47500 (C5), $67000(\mathrm{C} 18)$ and $88000(\mathrm{C} 50)$. A rather low molecular weight silicone prepolymer (P100) of viscosity $100 \mathrm{mPa} \cdot \mathrm{s}$ supplied by Petrarch Ltd. was also used. The catalyst, dioctyltinmaleate (DOTM) (LT195, M.W 459), was supplied by Lankro Ltd. and the crosslinker vinyltris(ethoxymethoxy)silane (VTEMS A172, M.W 280.4) Union Carbide Ltd., Lithium aluminum dinbutylamide was obtained from Lancaster Synthesis Ltd. as $0.16 \mathrm{M}$ solution in dimethoxyethane and was diluted to $0.1 \mathrm{M}$ before use as a titrant. Gold label anhydrous tetrahydrofuran (THF), 2-naphthol (99 percent pure) and phenylazodiphenylamine were obtained from Aldrich Chemical Co. Analar benzene and anhydrous dimethoxyethane from BDH Ltd. Barium oxide used as a drying agent was obtained from Hopkin and Williams Ltd.

\subsection{Hydroxyl $(\mathrm{OH})$ group determination of the prepolymers by titration}

Hydroxyl groups determination in silicone prepolymers has been carried out through titration according to an established analytical procedure $[6,7]$. About $25 \mathrm{ml}$ of dry THF were placed in a dry $50 \mathrm{ml}$ widemouth conical flask which contained a Teflon covered magnetic stirring bar. A few drops of 0.1 percent $(\mathrm{w} / \mathrm{v})$ solution of 4-phenylazodiphenyl amine in benzene (indicator solution) were added and the flask was sealed with a rubber septum. A slow stream of nitrogen dried by prior passage through a tube containing anhydrous $\mathrm{CaSO}_{4}$ was passed through the flask via a needle through the rubber septum. The magnetic stirrer was started and the hydroxyl groups present were titrated with $0.1 \mathrm{M}$ solution of lithium aluminum di-n-butyl amine in dimethoxyethane until the colour of the solution changed from yellow to red purple and persisted for $30 \mathrm{sec}$. Then an accurately weighed sample of the prepolymer $(0.3-0.5 \mathrm{~g})$ was syringed into the flask. The titrant was injected drop by drop into the flask via the rubber septum from a one $\mathrm{ml}$ syringe graduated in $0.01 \mathrm{ml}$ until the yellow to purple colour change persisted. The weight and volume of the titrant used for titration was noted. The concentration of $\mathrm{OH}$ groups was calculated using Equation (1) [6, 7]:

$\% \mathrm{OH}=\frac{V \cdot 0.017}{W_{1}} \cdot 100$

$V$ in the above equation is the volume $[\mathrm{ml}]$ and $W_{1}$ the weight $[\mathrm{g}]$ of the prepolymer used. The titrant was standardised with dry 2-naphthol (stored over barium oxide) using the above mentioned procedure and the normality $(N)$ of the titrant used was calculated as (Equation (2)):

$$
N=\frac{11.8 \cdot W_{2}}{V \cdot 0.017 \cdot 100}
$$

where 11.8 is the $\% \mathrm{OH}$ group in 2-naphthol and $W_{2}$ is the weight $[\mathrm{g}]$ of 2-naphthol.

The determinations of moisture content in the various prepolymers were carried out using the Karl Fischer method. The moisture content, converted to $\mathrm{OH}$ equivalence $(2 \mathrm{OH} / \mathrm{HO}=1.89)$, was subtracted from the total amount of $\mathrm{OH}$ groups determined for correction. The titration for each sample was

Table 1. Characterisation data of silicone prepolymers

\begin{tabular}{|c|c|c|c|c|c|c|c|c|}
\hline \multirow[b]{2}{*}{ Prepolymers } & \multirow{2}{*}{$\begin{array}{c}M_{n} \\
\text { (quoted) } \\
{[5]}\end{array}$} & \multicolumn{2}{|c|}{$\begin{array}{c}\mathbf{M}_{\mathbf{n}} \text { from viscometry } \\
\text { measurements }\end{array}$} & \multicolumn{2}{|c|}{ GPC Analysis } & \multicolumn{2}{|c|}{ OH Group determination } & \multirow{2}{*}{$\begin{array}{c}\overline{\mathbf{M}}_{\mathrm{c}} \text { from } \\
\text { swelling } \\
\text { measurements }\end{array}$} \\
\hline & & $\begin{array}{c}\text { Barry } \\
{[13]}\end{array}$ & $\begin{array}{c}\text { Wacker } \\
{[15]}\end{array}$ & $\mathbf{M}_{\mathbf{n}}$ & $\mathbf{M}_{\mathbf{w}}$ & $\% \mathrm{OH}$ & $\begin{array}{c}\mathrm{M}_{\mathrm{n}} \text { assuming } \\
\text { HOPDMS } \\
\text { bifunctional on } \% \mathrm{OH}\end{array}$ & \\
\hline P100 & 25000 & 4339 & 7145 & - & - & 1.094 & 2953 & 2250 \\
\hline $\mathrm{C} 1$ & 26714 & 31556 & 19967 & 20010 & 58000 & 0.162 & 19938 & 8500 \\
\hline $\mathrm{C} 2$ & 35850 & 40846 & 25004 & 17130 & 74880 & 0.139 & 22014 & 8600 \\
\hline C5 & 49984 & 55407 & 33957 & 27960 & 99130 & 0.138 & 23406 & 9000 \\
\hline C18 & 73673 & 74232 & 47749 & 36700 & 133400 & 0.105 & 30762 & 11500 \\
\hline $\mathrm{C} 50$ & 88000 & 93296 & 64670 & 45161 & 179250 & 0.085 & 38000 & 12000 \\
\hline
\end{tabular}


repeated three times and the average of these as the $\% \mathrm{OH}$ groups in the prepolymer chains are given in seventh column of the Table 1 .

\subsection{Viscosity measurements of prepolymers}

The viscosity measurements of the silicone prepolymers were carried out by Haake Rotovisco viscometer. About $10 \mathrm{ml}$ of prepolymer to be tested was poured into the sample cavity to fill to the inside mark. The sample cell was screwed up slowly to the bob. The gear lever was then engaged allowing different speed factors ranging 162-1 corresponding to a shear rate of $2.7-441 / \mathrm{sec}$. The corresponding viscosities (in $\mathrm{mPa} \cdot \mathrm{s}$ ) were then calculated.

\subsection{Swelling measurements of crosslinked samples}

For swelling measurements, each prepolymer has been cured with various amounts of crosslinker and the $\bar{M}_{c}$ determined for each cure according to the Flory-Rehner equation (Equation (3)) [8]:

$$
\bar{M}_{c}=\frac{-F_{f} \rho V_{1} v_{r}^{2 / 3} v_{2}^{1 / 3}}{\ln \left(1-v_{2}\right)+v_{2}+\chi v_{2}^{2}}
$$

where $F_{f}$ is a factor characterizing the extent to which the deformation in swelling approaches to affine limits. The details of the theoretical values of $F_{f}$ and that of its use as $F_{3}$ in the present case can found in the previous reports $[1,9,11-13]$. $V_{1}$ represents the molar volume of cyclohexane (105.87), $v_{r}$ is the volume fraction of polymer incorporated into the network (0.95), $v_{2}$ the volume fraction of polymer. $\chi$ is the cyclohexane polymer interaction parameter (0.42). $\rho$ is the density of the polymer (0.95).

The $\bar{M}_{c}$ limiting value for each prepolymer was obtained at the inflection point (from which onward the $\bar{M}_{c}$ values tends to become constant) from the respective plot of $\bar{M}_{c}$ vs. percent of crosslinker used for curing of that particular prepolymer. The measurement of for $\mathrm{C} 5$, using this procedure is given as an example in the form of Figure 1. The detailed procedure for $\bar{M}_{c}$ measurement is reported previously [9].

\subsection{Gel permeation chromatography (GPC)}

The gel permeation chromatography for all the prepolymers was carried out by using Shimadzu GPC calibrated with polystyrene standards. The columns used were Shimpack GPC-804, 802 and 802 having dimensions as $8.0 \mathrm{~mm}$ i.d. $\times 30 \mathrm{~cm}$ each. The operation temperature was $40^{\circ} \mathrm{C}$. Tetrahydrofuran was used as eluent and elution rate was set at $0.5 \mathrm{ml} / \mathrm{min}$. The detection was carried out by refractive index detector.

\section{Results}

\subsection{Characterisation of prepolymers}

Table 1 shows some molar masses characterisation data for the HOPDMS prepolymers used in this study. The number average molecular weights $\left(M_{n}\right)$ quoted by the manufacturer [5] are given in the second column. The physical techniques used for determining the molecular weights were bulk viscosity measurements (column 3 and 4), gel permeation chromatography (GPC) analysis (column 5 and 6) and swelling measurement (column 9). The number average molecular weights $\left(M_{n}\right)$ were calculated from the measured viscosities in $\mathrm{mPa} \cdot \mathrm{s}$ (at a shear rate $24.5 \mathrm{sec}$ ) from the following two calibration formulae (Equations (4) and (5)) [14, 15]:

$$
\log \eta=1+0.0123 M_{n}^{0.5}
$$

$\log \eta=3.08 \cdot \log M_{n}-10.06$

where $\eta$ - bulk viscosity of silicone fluid in centi stokes, $M_{n}$ - number average molecular weight, $m$ - molecular weight of the repeating unit (74) for PDMS.

Equation (4) is the Flory relationship [16] for melt viscosities confirmed for bulk viscosities of Polysiloxanes by Barry [14].

The Equation (5) is an empirical equation developed by Wacker-Chemie $\mathrm{GmbH}$ which is claimed [15] to give 'best fit' relation between melt viscosity and number average molecular weight.

The fifth column gives the number average molecular weights $\left(M_{n}\right)$ and the sixth column provides weight average molecular weights $\left(M_{w}\right)$ determined from the GPC analysis. The poldispersivity ranges from about 3 for $\mathrm{C} 1$ up to 4 for $\mathrm{C} 50$. The $\mathrm{OH}$ groups present in the polymer chain were measured by titrating the prepolymer with lithium aluminun 
n-butylamide. The percentage of water present in the prepolymer was measured by the Karl Fisher method and for correction subtracted from the total $\mathrm{OH}$ groups. The $\% \mathrm{OH}$ groups for the corresponding prepolymers can be seen in the seventh column of the Table 1. If it is assumed that all the prepolymers are bifunctional, the molecular weights $\left(M_{n}\right)$ based on the experimentally determined values of \%OH have been calculated (column 8) using Equation (6):

$M_{n}=\frac{34 \cdot 100}{\% \mathrm{OH}}\left[\frac{100-\% \text { cyclics }}{100}\right]$

where \%cyclics are percentage of soluble siloxanes that ranges from $D_{3}$ to $D_{8}$. The sol fraction for each weighed sample was obtained by extraction with cyclohexane. The weighed samples were put in cyclohexane for 15 days. During this period the solvent was changed once to facilitate the extraction of soluble materials. After removing the solvent, the specimens were dried to constant weight under vacuum at room temperature. The sol fraction for each sample was calculated. The values of sol fraction (about 5\% for all the Siloprenes except that for $\mathrm{C} 2$ where it was $10 \%$ ) obtained from each fully cured prepolymer network were used as \%cyclics in Equation (6).

The limiting values of $\bar{M}_{c}$ obtained from the swelling measurements for each prepolymers are provided in the ninth column. These $\bar{M}_{c}$ values represent the molecular weight between successive junctions when all the $\mathrm{OH}$ groups have satisfactorily reacted. The $\bar{M}_{c}$ value have been obtained at the inflection points (from which onward the $\bar{M}_{c}$ values tends to become constant) from the plot of

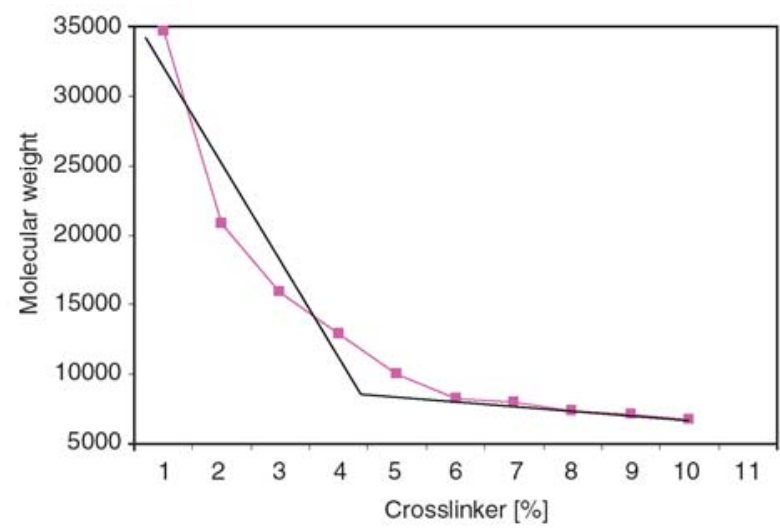

Figure 1. Variation of molecular weight between junctions for C5 with \% crosslinker
$\bar{M}_{c}$ values vs. percent of crosslinker used for curing the corresponding prepolymer. The $\bar{M}_{c}$ determination at the inflection point for C5 is shown in Figure 1. The limiting values for Wacker prepolymer (viscosity $50 \mathrm{~Pa} \cdot \mathrm{s}$ ) networks obtained as above from swelling measurements were found to be the same as that of for $\bar{M}_{c}$ the C50 networks.

Comparing the molecular weights, it is clear that $M_{n}$ values from the viscosity calibration Equation (4) approximate to the Bayer quoted values for $M_{n}$. The $M_{n}$ values from GPC though consistent with that obtained from the $\mathrm{OH}$ determination differed not only from the quoted values but also from $\bar{M}_{c}$. All the values of $M_{n}$ were much higher than the corresponding $\bar{M}_{c}$ and for the prepolymers used (C1-C50) in this study, the differences ranged 3-7 folds.

\section{Discussion}

\subsection{Difficulties in characterisation of prepolymers}

The paucity of $\mathrm{OH}$ groups on the polymer chains and the nature of spectroscopic methods such as IR (infrared) and NMR (nuclear magnetic resonance) have made it difficult to characterise the prepolymers used in this study by this technique. The results of characterisation of prepolymers by methods such as GPC, OH group titration, bulk viscosity and swelling measurements with the exception of viscosity measurements do not agree with the manufacturer's quoted number average molecular weights (Table 1).

The dissimilarity between the various $M_{n}$ values determined for the prepolymers used can be attributed to: the diverse nature, sensitivity, accuracy and limitation of the different experimental techniques used. The GPC separates molecules according to their effective size; it may not adequately distinguish between branched and linear polymers of the same molecular weight [17]. With the HOPDMS analysis the pendant $\mathrm{OH}$ group present along the chain will presumably make it impossible for the GPC to differentiate this chain from that of a linear chain without this group. The $\mathrm{OH}$ group titration gives the number of functional groups per specific amount of polymer or prepolymer without any indication about their molecular weight distribution. For linear polymer, it is possible to assume that the total number of chain ends is twice the number of 
polymer molecules. However if branching exist, then this assumption gives erroneous results in respect of estimating the number average molar mass.

The viscosity measurements for all the prepolymers were carried out at different shear rate range of $2.7-441 / \mathrm{sec}$. During these measurements, each of the materials behaved almost like Newtonian fluid. However, the molecular weights for each material was calculated for the viscosities determined at on shear rate i.e. $24.5 / \mathrm{sec}$ so that comparison can be made between them. The melt viscosity though used for $M_{n}$ determination is also sensitive to weight average molecular weight [17]. The different $M_{n}$ for each prepolymer (Table 1) obtained using independent viscosity calibration equations reflects the uncertainty relating melt viscosity to the number average molecular weight. Thus the results of the molecular weight determination through these different techniques would not be expected to be consistent. The similarity of the quoted [5] $M_{n}$ and that calculated through viscosity measurement (using Equation (4)) when compared with that from the other techniques showed disparities. It is likely that the quoted number average molecular weights are in fact the average molecular weights obtained through viscosity measurements (using Equation (4)); they therefore are misleading. The manufacturer's information seemed to imply that the prepolymer samples were essentially terminally bifunctional in $\mathrm{OH}$ and moreover search of the published literature never revealed any reference to multifunctionality in this type of silicone prepolymers. The $\bar{M}_{c}$ values of the silicone polymer networks crosslinked with low amounts of crosslinker or catalyst were somewhat near to the $M_{n}$ from the GPC analysis and $\mathrm{OH}$ group titration. However, as reported $[9,10,18]$, these networks are partially cured and so these values represent the networks where the $\mathrm{OH}$ groups have not sufficiently reacted.

Stochiometric amounts of crosslinker calculated on the basis of molar masses of the prepolymers assuming bifunctionality, does not cure the material. On the other hand the stoichiometric amounts of crosslinker calculated on the $\bar{M}_{c}$ basis corresponding theoretically to the point where all the $\mathrm{OH}$ groups have reacted gives fully cured polymer networks. Comparing the values of $\bar{M}_{c}$ (Table 1) for each prepolymer network, it is surprising that with the exception of P100 they vary so little, bearing in mind the large variation in molecular weight of the silicones from which they were prepared. The values of $M_{n}$ obtained through OH determination which is comparatively smaller than the Bayer quoted values support this view. If the prepolymers were strictly bifunctional, the $M_{n}$ from the $\mathrm{OH}$ determination should have been equal to the quoted number average molecular weights. In fact, the molecular weights determined from the $\mathrm{OH}$ determinations are in agreement with the values from GPC but are higher than the $\bar{M}_{c}$.

In order to determine the number of functional groups per chain, each molecular weight $\left(M_{n}\right)$ in turn was divided by the $\bar{M}_{c}$ values. This data is presented in Table 2. Assuming the $\mathrm{OH}$ group titration and the $\bar{M}_{c}$ data on the materials reliable, the functionality of the prepolymer was calculated as $\left(M_{n} / \bar{M}_{c}\right)+1$. These values are given in the last column of the Table 2. The number of functional groups calculated in this way for all the silicone prepolymer (last column of Table 2) suggests that irrespective of the prepolymer the ratio of the $\mathrm{OH}$ groups per unit chain length is approximately constant.

Table 2. Molecular weight of various prepolymers in multiple of $M_{c}$

\begin{tabular}{|c|c|c|c|c|c|c|c|c|}
\hline \multirow[t]{2}{*}{ Prepolymers } & \multirow{2}{*}{$\stackrel{M_{n}}{\text { (quoted) [5] }}$} & \multicolumn{2}{|c|}{$\begin{array}{c}\mathbf{M}_{\mathbf{n}} \text { from viscometry } \\
\text { measurements }\end{array}$} & \multicolumn{2}{|c|}{ GPC analysis } & \multirow{2}{*}{$\begin{array}{c}M_{n} \\
\text { from \%OH }\end{array}$} & \multirow[t]{2}{*}{$\overline{\mathbf{M}}_{\mathrm{c}}$} & \multirow{2}{*}{$\begin{array}{c}\text { Functionalities* } \\
\qquad\left(\mathbf{M}_{\mathbf{n}} / \overline{\mathbf{M}}_{\mathbf{c}}\right)+\mathbf{1}\end{array}$} \\
\hline & & Barry [13] & Wacker [15] & $\mathbf{M}_{\mathbf{n}}$ & $\mathbf{M}_{\mathbf{w}}$ & & & \\
\hline P100 & - & 1.930 & 3.175 & - & - & 1.38 & 2250 & 2.31 \\
\hline $\mathrm{C} 1$ & 3.14 & 3.712 & 2.349 & 2.35 & 6.824 & 2.47 & 8500 & 3.35 \\
\hline $\mathrm{C} 2$ & 4.17 & 4.750 & 2.907 & 1.99 & 8.707 & 2.84 & 8600 & 3.56 \\
\hline $\mathrm{C} 5$ & 5.55 & 6.156 & 3.773 & 3.11 & 11.01 & 2.74 & 9000 & 3.60 \\
\hline C18 & 6.41 & 6.455 & 4.152 & 3.19 & 11.6 & 2.82 & 11500 & 3.67 \\
\hline C50 & 7.33 & 7.775 & 5.389 & 3.76 & 14.94 & 3.33 & 12000 & 4.17 \\
\hline
\end{tabular}

*Based on the $\mathrm{OH}$ titration $\left(M_{n}\right)$ and $\bar{M}_{c}$ 


\section{Conclusions}

In view of the method of preparation of the silicone prepolymer from chloro silanes and the difficulty of separating dichloro and trichloro silanes due to the close boiling points i.e. $70,66^{\circ} \mathrm{C}$, it follows that the preparation of solely linear hydroxy terminated bifunctional PDMS is unlikely and that irrespective of the molecular weight of the prepolymers the number of silanol groups per unit chain length may not differ significantly. For example, the presence of one mole of trichlorodimethylsilane during the preparation of polydimethylsiloxanes will decrease the degree of polymerisation to only 200 on the completion of reaction [19] and the resulting polymer will have pendant groups or branching.

The unexpected result was the insensitivity of the properties to the initial molar mass of the polymers from which the networks was derived. The reason for this was the multifuntionality of the prepolymers, a characteristic not previously suspected or reported.

Whilst there is this molecular uncertainty it is the $\bar{M}_{c}$ values which represent the actual structure of the cured silicone elastomers and which dictate the properties of the polymer network under stress conditions. It is for this reason that the respective $\bar{M}_{c}$ values must be used for the interpretation of the results of any mechanical properties of the polymer networks.

\section{References}

[1] Wake W. C., Tidd B. K., Loadman M. J. R.: Analysis of rubbers and rubber-like polymers. Applied Science Publishers, London (1983).

[2] Baijal M. D.: Application of metallic membrane filters in the clarification of styrene-butadiene rubber solutions for gel permeation chromatography. Analytical Chemistry, 44, 1337-1338 (1972).

[3] Barrere M., Ganachaud F., Bendejacq D., Dourges M. A., Maitre C., Henery P.: Anionic polymerization of octamethylcyclotetrasiloxane in miniemulsion. II Molar masses analyses and mechanism scheme. Polymer, 42, 7239-7246 (2001).
[4] Kazmierski K., Hurduc N., Sauvet G., Chojnowski J.: Polysiloxanes with chlorobenzyl groups as precursors of new organic-silicone materials. Journal of Polymer Science Part A: Polymer Chemistry, 42, 1682-1692 (2004).

[5] Product brochure of Bayer. Bayer AG, Germany (1983).

[6] Kellum G. E., Uglum K. L.: Lithium aluminum dibutylamide as a direct acidbase titrant for determination of silanols. Analytical Chemistry, 39, 1623-1627 (1967).

[7] Smith A. L.: Analysis of silicones. Wiley, New York (1974).

[8] Flory P. J., Erman B.: Theory of elasticity of polymer networks. 3. Macromolecules, 15, 800-806 (1982).

[9] Shah G. B., Winter W.: Effect of bimodality on tear properties of silicone networks. Macromolecular Chemistry and Physics, 197, 2201-2208 (1996).

[10] Shah G. B.: Aspects of the polycondensation of hydroxyfunctional polydimethylsiloxanes. $\mathrm{PhD}$ Thesis, UWCC (UK) (1990).

[11] Flory P. J., Gordon M., McCrum N. G.: Statistical thermodynamics of random networks [and discussion]. Proceedings of the Royal Society of London. Series A, Mathematical and Physical Sciences, 351, 351-380 (1976).

[12] Flory P. J.: The elastic free energy of dilation of a network. Macromolecules, 2, 119-122 (1979).

[13] Flory P. J.: Theory of elasticity of polymer networks. The effect of local constrain on junctions. Journal of Chemical Physics, 66, 5720-5729 (1977).

[14] Barry A. J.: Viscometric investigation of dimethylsiloxane polymer. Journal of Applied Physics, 17, 1020-1024 (1946).

[15] Private Communication Wacker-Chemie GmbH: The relationship between molecular weight and bulk viscosity. pp. 1-3, 20 $0^{\text {th }}$ September (1988).

[16] Flory P. J.: Viscosities of linear polyesters. An exact relationship between viscosity and chain length. Journal of the American Chemical Society, 62, 1057-1070 (1940).

[17] White J. R., Campbell D.: Polymer characterisation physical techniques. Chapman and Hall, London (1989).

[18] Shah G. B.: Bimodality and increased amount of crosslinker enhances tensile properties of silicone networks. e-Polymers, no. 113 (2007).

[19] Mark H. F., Tobolsky A. V.: Physical chemistry of high polymeric systems. Interscience, New York (1950). 